\title{
ON THE EINSTEIN-KÄHLER METRIC AND THE HOLONOMY OF A LINE BUNDLE
}

\author{
KENJI TSUBOI \\ Laboratory of Mathematics, Tokyo University of Fisheries, \\ 4-5-7 Kohnan, Minato, Tokyo 108-8477, Japan
}

(Received 29 March 2000)

\begin{abstract}
In this paper we give a relation between the Futaki invariant for a compact complex manifold $M$ and the holonomy of a determinant line bundle over a loop in the base space of any principal $G$ bundle, where $G$ is the identity component of the maximal compact subgroup of the complex Lie group consisting of all biholomorphic automorphisms of $M$. Using the property of the Futaki invariant, we show that the holonomy is an obstruction to the existence of the Einstein-Kähler metrics on $M$. Our main result is Theorem 2.1 .
\end{abstract}

Keywords: Einstein-Kähler metric; holonomy; Futaki invariant; eta invariant; index theorem

AMS 2000 Mathematics subject classification: Primary 32Q20

Secondary 58J28; 58J52

\section{Introduction}

Let $M$ be a compact connected complex $m$-dimensional manifold, $H(M)$ the finitedimensional complex Lie group consisting of all biholomorphic automorphisms of $M$, and $h(M)$ its Lie algebra consisting of all holomorphic vector fields on $M$. A Kähler metric with Kähler form $\omega$ is called an Einstein-Kähler metric if the Ricci form of $\omega$ is a constant multiple of $\omega$. In [4] Futaki defined a Lie algebra homomorphism $f: h(M) \rightarrow \boldsymbol{C}$, which is called the Futaki invariant, and proved that $f(X)=0$ for any $X \in h(M)$ if $M$ admits an Einstein-Kähler metric. Let $\nabla$ be a type $(1,0)$ connection of the holomorphic tangent bundle $T M$ with its connection form $\theta$ and $L(X)$ the $g l(m ; \boldsymbol{C})$-valued 0-form defined by $L(X)=L_{X}-\nabla_{X}$ for $X \in h(M)$. Then, by multiplying a constant factor to the Futaki invariant in [4], the Futaki invariant is expressed as follows:

$$
f(X)=\int_{M} c_{1}^{m+1}\left(\frac{\mathrm{i}}{2 \pi}(L(X)+\Theta)\right)
$$

where $c_{1}$ is the first Chern polynomial and $\Theta$ is the curvature form of $\theta$ (see [5, Proposition 2.3]).

Now let $G$ be the identity component of the maximal compact subgroup of $H(M), \vartheta$ its Lie algebra, and $\pi: P \rightarrow B$ any principal $G$-bundle over a smooth manifold $B$ with a 
connection. Let $h$ be any $G$-invariant Hermitian metric of $T M$. Then we can assume that the type $(1,0)$ connection $\nabla$ is a $G$-invariant unitary connection. Let $L$ be the virtual holomorphic $G$-bundle over $M$ defined by

$$
L=\otimes^{m+1}\left(K_{M}^{-1}-\tau\right),
$$

where $K_{M}^{-1}$ is the anticanonical bundle of $M$ and $\tau$ is the trivial complex line bundle over $M$ with the trivial $G$-action. Then the metric $h$ and the standard metric of $\tau$ define a $G$-invariant metric $h^{L}$ of $L$, and the connection $\nabla$ and the trivial connection of $\tau$ define a $G$-invariant unitary connection $\nabla^{L}$ of $L$. Moreover, since the complex manifold $M$ has the natural spin ${ }^{c}$-structure, the half spinor bundles $S^{ \pm}$on $M$ are defined and the $L$-valued $\operatorname{spin}^{\mathrm{c}}$-Dirac (Dolbeault) operator,

$$
D^{L}: \Gamma\left(S^{+} \otimes L\right) \rightarrow \Gamma\left(S^{-} \otimes L\right),
$$

on $M$ is defined by using the metrics $h, h^{L}$ and the connections $\nabla, \nabla^{L}$. Here we can define a smooth fibration of manifolds $F \rightarrow B$ with fibre $M$ by $F=P \times_{G} M$. Since $G$ preserves all structures defining $D^{L}$, we can define a locally constant family of spin ${ }^{\mathrm{c}}$-Dirac operators $D^{F}:=P \times_{G} D^{L}$ parametrized by $B$. Let $\zeta$ be the determinant line bundle of the family $D^{F}$. Then it is clear that $\zeta=P \times_{G} K$, where $K$ is a one-dimensional complex $G$-module defined by $K=\wedge^{k}\left(\left(\operatorname{ker} D^{L}\right)^{*}\right) \otimes \wedge^{l} \operatorname{ker}\left(\left(D^{L}\right)^{*}\right)$, where $k$ and $l$ are the dimensions of $\operatorname{ker} D^{L}$ and $\operatorname{ker}\left(\left(D^{L}\right)^{*}\right)$, respectively. Hence, the connection in $P$ defines the connection of $\zeta$ and the holonomy $\operatorname{hol}_{\zeta}(\gamma)$ of $\zeta$ around any loop $\gamma$ in $B$ is defined.

\section{Main result}

The next theorem is our main result.

Theorem 2.1. Let $\gamma$ be any loop in $B$ and $b$ any point on $\gamma$. Assume that a horizontal lift of $\gamma$ in $P$ connects a point $p \in \pi^{-1}(b)$ with the point $p \exp X \in \pi^{-1}(b)$ for $X \in \vartheta$. Then the following equality holds:

$$
\operatorname{hol}_{\zeta}(\gamma)=\mathrm{e}^{-2 \pi \mathrm{i} f(X)} .
$$

Proof. The strategy for the proof is as follows. First we will give a relation between the holonomy $\operatorname{hol}_{\zeta}(\gamma)$ and the eta invariant of $M \times S^{1}$ with respect to a metric corresponding to the holonomy by using Witten's holonomy formula. Then we will show that the Futaki invariant $f(X)$ is equal to the integral of the Chern form on $M \times D^{2}$ whose boundary is $M \times S^{1}$ by means of direct calculation. We will finally show that the eta invariant is equal to the integral by using the Atiyah-Patodi-Singer Theorem.

First note that $f(X) \in \boldsymbol{R}$. We can demonstrate this fact as follows. Since both the $G$-action and the connection $\nabla$ preserve $h$, it follows that $L_{X} h=0$ and $\nabla_{X} h=0$. Hence it follows that $L(X) h=0$, and therefore $L(X)$ is skew-Hermitian with respect to the metric $h$ and has only pure imaginary eigenvalues, as does $\Theta$. Hence it follows that

$$
f(X)=\int_{M} c_{1}^{m+1}\left(\frac{\mathrm{i}}{2 \pi}(L(X)+\Theta)\right) \in \boldsymbol{R} .
$$


Now let $S^{1}=\boldsymbol{R} / \boldsymbol{Z}$ be the circle with coordinate $t(0 \leqslant t \leqslant 1), g_{0}$ the metric on $S^{1}$ which comes from the standard metric on $\boldsymbol{R}, W$ the product space $W=M \times S^{1}$, and $q_{S}: W \rightarrow S^{1}$ the natural projection. Then the horizontal subspace $q_{S}^{*} T S^{1}$ of the fibration $q_{S}: W \rightarrow S^{1}$, which is different from the obvious horizontal subspace, is defined by the vector field $Y:=X+(\partial / \partial t)$, where $X \in \vartheta$ is identified with the real vector field corresponding to $X$. Hence we can define the product Riemannian metric of $T W=$ $T M \oplus q_{S}^{*} T S^{1}$ by $h \oplus\left(g_{0} / \varepsilon^{2}\right)$, where $\varepsilon$ is an arbitrary positive constant. Let $\zeta^{W}$ be the determinant line bundle of the trivial family $D^{L} \times S^{1}$ parametrized by $S^{1}$, and let $\operatorname{hol}_{\zeta^{W}}\left(S^{1}\right)$ be the holonomy of $\zeta^{W}$ around $S^{1}$ with respect to the connection in $W$ defined by $q_{S}^{*} T S^{1}$. Since the horizontal curve $\tilde{\gamma}=\{(\exp s X \cdot p, b+s) \mid 0 \leqslant s \leqslant 1\}$ in $W$ connects the point $(p, b)$ with the point $(\exp X \cdot p, b)$ for any point $(p, b) \in W\left(p \in M, b \in S^{1}\right)$, $\operatorname{hol}_{\zeta^{W}}\left(S^{1}\right)$ equals $\left.\exp X\right|_{K} \in C$, which coincides with $\operatorname{hol}_{\zeta}(\gamma)$. Hence it follows that

$$
\operatorname{hol}_{\zeta}(\gamma)=\operatorname{hol}_{\zeta^{W}}\left(S^{1}\right)
$$

Here the connections $\nabla, \nabla^{L}$ and the horizontal subspace $q_{S}^{*} T S^{1}$ define unitary connections $\nabla^{W}$ of $T W$ and $\nabla^{\prime}$ of the virtual bundle $L^{W}:=L \times S^{1}$ over $W$. Let $A_{\varepsilon}^{W}$ be the $L^{W}$-valued self-adjoint Dirac operator on $W$ defined by using the connections $\nabla^{W}$, $\nabla^{\prime}$, the metric $h \oplus\left(g_{0} / \varepsilon^{2}\right)$, and the $\operatorname{spin}^{\mathrm{c}}$-structure of $T W$ defined by the natural spin ${ }^{\mathrm{c}}$ structure of $T M$ and the unique trivial spin ${ }^{\mathrm{c}}$-structure of $T S^{1}, \eta_{\varepsilon}^{W}$ the eta invariant of $A_{\varepsilon}^{W}, d_{\varepsilon}^{W}:=\operatorname{dim} \operatorname{ker} A_{\varepsilon}^{W}$ and $\xi_{\varepsilon}^{W}:=\frac{1}{2}\left(\eta_{\varepsilon}^{W}+d_{\varepsilon}^{W}\right)$. Then the next equality follows from the Witten's holonomy formula [3, Theorem 3.16]:

$$
\operatorname{hol}_{\zeta^{W}}\left(S^{1}\right)=\lim _{\varepsilon \rightarrow+0}(-1)^{\operatorname{Index}\left(D^{L}\right)} \mathrm{e}^{-2 \pi \mathrm{i} \xi_{\varepsilon}^{W}},
$$

where $\operatorname{Index}\left(D^{L}\right)$ is the Atiyah-Singer index of $D^{L}$.

Let $\theta^{W}$ denote the connection form of $\nabla^{W}$. Then we can see that

$$
\theta^{W}=q_{W}^{*} \theta+L(X) \mathrm{d} t
$$

where $q_{W}: W \rightarrow M$ is the natural projection because

$$
\nabla_{\partial / \partial t}^{W} Z=\nabla_{Y}^{W} Z-\nabla_{X}^{W} Z=\frac{\mathrm{d}}{\mathrm{d} s}\left[\exp (-s X)_{*} Z\right]_{s=0}-\nabla_{X} Z=L(X)(Z)
$$

for any $Z \in h(M)$. Now let $I=[1,2]$ be an interval with coordinate $r, C$ the cylinder defined by $C=I \times S^{1}=\{(r, t) \mid 1 \leqslant r \leqslant 2,0 \leqslant t \leqslant 1\}$, and $V$ the product space $V=M \times C$. Then the boundary of $V$ consists of two components $W_{1}=M \times\{1\} \times S^{1}$ and $W_{2}=M \times\{2\} \times S^{1}$. Let $\varphi(r)$ be a smooth function such that $0 \leqslant \varphi(r) \leqslant 1, \varphi(r)=0$ for $r \in\left[1, \frac{4}{3}\right], \varphi(r)=1$ for $r \in\left[\frac{5}{3}, 2\right]$, and $\left(z_{1}, z_{2}, \ldots, z_{m}\right)$ a local holomorphic coordinate on $M$. Let $Y^{r}$ denote the vector field on $V$ defined by

$$
Y^{r}=\varphi(r) X+\frac{\partial}{\partial t}
$$


Then a complex structure $J^{V}$ and a Hermitian metric $h^{V}$ on $V$ is defined by using the complex structure $J$ and the Hermitian metric $h$ on $M$ as follows:

$$
\begin{gathered}
J^{V}\left(\frac{\partial}{\partial z_{i}}\right)=J\left(\frac{\partial}{\partial z_{i}}\right), \quad J^{V}\left(\frac{\partial}{\partial r}\right)=\varepsilon Y^{r} \\
h^{V}\left(\frac{\partial}{\partial z_{i}}, \frac{\partial}{\partial z_{j}}\right)=h\left(\frac{\partial}{\partial z_{i}}, \frac{\partial}{\partial z_{j}}\right), \quad h^{V}\left(\frac{\partial}{\partial r}, \frac{\partial}{\partial r}\right)=\varepsilon^{2} h^{V}\left(Y^{r}, Y^{r}\right)=1, \\
h^{V}\left(\frac{\partial}{\partial z_{i}}, \frac{\partial}{\partial r}\right)=h^{V}\left(\frac{\partial}{\partial z_{i}}, Y^{r}\right)=h^{V}\left(\frac{\partial}{\partial r}, Y^{r}\right)=0 .
\end{gathered}
$$

Then we can define a unitary connection $\theta^{V}$ of $T V$ by

$$
\theta^{V}=q_{V}^{*} \theta+\varphi(r) L(X) \mathrm{d} t
$$

where $q_{V}: V \rightarrow M$ is the natural projection. Note that the restrictions of the metric and the connection on $V$ to $W_{2}$ coincide with those of $W$ and hence $W_{2}$ is identified with $W$. Now the curvature form $\Theta^{V}$ of $\theta^{V}$ is computed as

$$
\Theta^{V}=\mathrm{d} \theta^{V}+\theta^{V} \wedge \theta^{V}=q_{V}^{*} \Theta+\varphi^{\prime}(r) L(X) \mathrm{d} r \wedge \mathrm{d} t \quad\left(\bmod \mathrm{d} z_{i} \wedge \mathrm{d} t\right)
$$

and hence it follows from (1.1) that

$$
\begin{aligned}
\int_{V} c_{1}^{m+1}\left(T V, \Theta^{V}\right) & =\int_{V}\left(\operatorname{Tr}\left(\frac{\mathrm{i}}{2 \pi} \Theta^{V}\right)\right)^{m+1} \\
& =\int_{M}(m+1)\left(\operatorname{Tr}\left(\frac{\mathrm{i}}{2 \pi} \Theta\right)\right)^{m} \operatorname{Tr}\left(\frac{\mathrm{i}}{2 \pi} L(X)\right) \int_{1}^{2} \varphi^{\prime}(r) \mathrm{d} r \int_{0}^{1} \mathrm{~d} t \\
& =\int_{M} c_{1}^{m+1}\left(\frac{\mathrm{i}}{2 \pi}(L(X)+\Theta)\right)=f(X)
\end{aligned}
$$

where $c_{1}\left(T V, \Theta^{V}\right)$ is the first Chern form with respect to $\Theta^{V}$.

Now let $U$ be the product space $U=M \times D^{2}$. Then the product complex structure of the complex structures on $M$ and $D^{2}$ define a complex structure on $U$, which coincides with $J^{V}$ near the boundary $\partial U=W_{1}$. We give a rotationally symmetric Hermitian metric $h^{D}$ on $D^{2}$ which is a product metric of $(1-\delta, 1] \times S^{1}$ near the boundary $\partial D^{2}=\{1\} \times S^{1}=$ $S^{1}$, where the metric on $S^{1}$ is $g_{0} / \varepsilon^{2}$. Let $\theta^{D}$ be the type $(1,0)$ unitary connection of $T D^{2}$ and $\Theta^{D}$ its curvature form. Then the product metric of $h$ and $h^{D}$ define a Hermitian metric $h^{U}$ on $U$, which coincides with $h^{V}$ near $W_{1}$, and the direct sum of $\theta$ and $\theta^{D}$ define a unitary connection $\theta^{U}$ of $T U$, which coincides with $\theta^{V}$ near $W_{1}$. Let $N$ denote the complex manifold with boundary $W_{2}=W$ defined by gluing $U$ to $V$ along the boundary $W_{1}$. Then the metrics $h^{V}, h^{U}$ and the connections $\theta^{V}, \theta^{U}$ define a Hermitian metric $h^{N}$ and a unitary connection $\theta^{N}$ of $T N$. We denote by $\Theta^{U}, \Theta^{N}$ the curvature forms of $\theta^{U}$, $\theta^{N}$, respectively. Let $c_{1}(T M)$ be the first Chern class of $T M$ and $[M]$ the fundamental 
cycle of $M$. Then it follows from (2.4) that

$$
\begin{aligned}
\int_{N} c_{1}^{m+1}\left(T N, \Theta^{N}\right) & =\int_{V} c_{1}^{m+1}\left(T V, \Theta^{V}\right)+\int_{U} c_{1}^{m+1}\left(T U, \Theta^{U}\right) \\
& =f(X)+(m+1) c_{1}^{m}(T M)[M] \int_{D^{2}} c_{1}\left(T D^{2}, \Theta^{D}\right) \equiv f(X) \quad(\bmod \boldsymbol{Z}) .
\end{aligned}
$$

On the other hand, let $L^{N}$ be a virtual bundle over $N$ defined by $L^{N}=\otimes^{m+1}\left(K^{-1} N-\right.$ $\tau)$. Then the metric $h^{N}$ and the connection $\theta^{N}$ naturally define a metric and a unitary connection of $L^{N}$, whose restriction to $\partial N=W$ coincides with those of $L^{W}=\left.L^{N}\right|_{W}$. Hence, using the metrics, the connections and the natural spinc-structure of $T N$, we can define the $L^{N}$-valued spin ${ }^{c}$-Dirac operator as in the previous section. Then since the restrictions of the metric $h^{N}$ and the connection $\theta^{N}$ to $\partial N=W$ coincide with those of $T W$ and $h^{N}, \theta^{N}$ are products near $W$, it follows from the Atiyah-Patodi-Singer Index Theorem (see $[\mathbf{2},(4.2)]$ and $[\mathbf{1},(4.3)])$ that

$$
\int_{N} \operatorname{ch}\left(L^{N}, \Theta^{N}\right) \operatorname{Td}\left(T N, \Theta^{N}\right) \equiv \xi_{\varepsilon}^{W} \quad(\bmod \boldsymbol{Z}),
$$

where $\operatorname{ch}\left(L^{N}, \Theta^{N}\right)$ is the Chern character form of $L^{N}$ and $\operatorname{Td}\left(T N, \Theta^{N}\right)$ is the Todd form of $T N$. Here, since

$$
\operatorname{ch}\left(L^{N}, \Theta^{N}\right)=\left\{\operatorname{ch}\left(\wedge^{m+1} T N, \Theta^{N}\right)-1\right\}^{m+1}=c_{1}^{m+1}\left(T N, \Theta^{N}\right)
$$

and the leading term of $\operatorname{Td}(T N)$ is equal to 1, it follows from (2.5) and (2.6) that

$$
f(X) \equiv \xi_{\varepsilon}^{W} \quad(\bmod \boldsymbol{Z}) .
$$

Moreover, since the Futaki invariant does not depend on the choice of $\varepsilon$, it follows from $(2.2),(2.3)$ and $(2.7)$ that

$$
\operatorname{hol}_{\zeta}(\gamma)=(-1)^{\operatorname{Index}\left(D^{L}\right)} \mathrm{e}^{-2 \pi \mathrm{i} f(X)} .
$$

Here it follows from the Atiyah-Singer Index Theorem (see [1, (4.3)]) that

$$
\begin{aligned}
\operatorname{Index}\left(D^{L}\right) & =\operatorname{ch}(L) \operatorname{Td}(T M)[M] \\
& =\left(c_{1}(T M)+\text { higher-order terms }\right)^{m+1}(1+\cdots)[M]=0,
\end{aligned}
$$

and hence it follows from (2.8) that

$$
\operatorname{hol}_{\zeta}(\gamma)=\mathrm{e}^{-2 \pi \mathrm{i} f(X)} .
$$

This completes the proof of Theorem 2.1.

The next corollary is an immediate consequence of Theorem 2.1 and the main theorem in $[4]$. 
Corollary 2.2. $\operatorname{hol}_{\zeta}(\gamma)=1$ for any loop $\gamma$ in $B$ if $M$ admits an Einstein-Kähler metric.

Remark 2.3. Let $\pi: E G \rightarrow B G$ be the universal $G$-bundle with the universal connection. Then, for any $g=\exp X \in G$, there exists a loop $\gamma_{g}$ in $B G$ such that a horizontal lift of $\gamma_{g}$ connects a point $p \in \pi^{-1}(b)$ with the point $p \cdot g \in \pi^{-1}(b)$. Hence it follows from Theorem 2.1 that $\operatorname{hol}_{\zeta}(\gamma)=1$ for any loop $\gamma$ in $B G$ if and only if $f(X) \in \boldsymbol{Z}$ for any $X \in \vartheta$, which is equivalent to the condition that $f(X)=0$ for any $X \in \vartheta$. On the other hand, it is known (see $[\mathbf{6}$, Theorems 5.1, 5.2]) that $M$ does not admit an Einstein-Kähler metric unless the Lie algebra $h(M)$ coincides with the complexification of $\vartheta$ or $\vartheta$ itself. If $h(M)$ coincides with the complexification of $\vartheta$ or $\vartheta$ itself, $f(X)=0$ for any $X \in \vartheta$ if and only if $f(X)=0$ for any $X \in h(M)$.

Let $\vartheta_{p}$ be the $A d(G)$-invariant dense subset of $\vartheta$ consisting of the elements $X$ such that $\exp X$ is periodic and $\Omega_{p}(B)$ the set of loops in $B$ whose horizontal lifts connect a point $p \in \pi^{-1}(b)$ with the point $p \cdot \exp (X) \in \pi^{-1}(b)$ for $X \in \vartheta_{p}$. Then the next proposition follows from [7, Theorem 1.4] and (2.9).

Proposition 2.4. Assume that $\gamma$ is an element of $\Omega_{p}(B)$. Then the following equality holds:

$$
\operatorname{hol}_{\zeta}(\gamma)=\exp \frac{2 \pi \mathrm{i}}{p} \sum_{k=1}^{p-1} \frac{1}{\mathrm{e}^{-2 \pi \mathrm{i} k / p}-1} \operatorname{Index}\left(D^{L}, g^{k}\right),
$$

where $p$ is the order of $g:=\exp X$ and $\operatorname{Index}\left(D^{L}, g^{k}\right)$ is the Atiyah-Singer index of $D^{L}$ evaluated at $g^{k}$ (see [1]).

Since $\operatorname{Index}\left(D^{L}, g^{k}\right)$ is computed by using the holomorphic Lefschetz theorem (4.6) in $[\mathbf{1}]$, the holonomy $\operatorname{hol}_{\zeta}(\gamma)$ is computed concretely for $\gamma \in \Omega_{p}(B)$.

Example 2.5. In this example we compute the holonomy for the complex manifold introduced in [4]. Let $H_{i}$ denote the hyperplane bundle over $\boldsymbol{C} \boldsymbol{P}^{i}$ and $M$ the total space of the projective bundle $\boldsymbol{P}(E)$ of the vector bundle $E=\pi_{1}^{*} H_{1} \oplus \pi_{2}^{*} H_{2}$ over $\boldsymbol{C P}^{1} \times \boldsymbol{C P}^{2}$, where $\pi_{i}$ is the $i$ th factor projection. Then the factor group $P(\mathrm{GL}(2 ; \boldsymbol{C}) \times \mathrm{GL}(3 ; \boldsymbol{C}))$ of $\mathrm{GL}(2 ; \boldsymbol{C}) \times \mathrm{GL}(3 ; \boldsymbol{C})$ by the centre of $\mathrm{GL}(5 ; \boldsymbol{C})$ is isomorphic to the identity component of $H(M)$, hence $h(M)$ is the complexification of $\vartheta$, and $M$ does not admit an EinsteinKähler metric (for details, see $[\mathbf{4}, \S 3]$ ). Now let $\pi: E G \rightarrow B G$ be the universal $G$-bundle with the universal connection. Then, for any $g \in G$ there exists a loop $\gamma_{g}$ in $B G$ such that a horizontal lift of $\gamma_{g}$ connects a point $p \in \pi^{-1}(b)$ with the point $p \cdot g \in \pi^{-1}(b)$. Here let $X$ be an element of $\vartheta_{p}$ represented by the diagonal matrix with diagonal entries $(2 \pi \mathrm{i} / p, 2 \pi \mathrm{i} / p, 0,0,0)$ and set $g:=\exp X$, which is an element of $P(\operatorname{GL}(2 ; \boldsymbol{C}) \times \operatorname{GL}(3 ; \boldsymbol{C}))$ represented by the diagonal periodic matrix of order $p$ with diagonal entries $(\alpha, \alpha, 1,1,1)$, where $\alpha:=\exp (2 \pi \mathrm{i} / p)$ is the primitive $p$ th root of 1 . Then the fixed-point set $\Omega(k) \subset M$ of the $g^{k}$-action is independent of $k$ and coincides with the disjoint union of the two components $N_{1}, N_{2}$, which are isomorphic to the base space $\boldsymbol{C} \boldsymbol{P}^{1} \times \boldsymbol{C} \boldsymbol{P}^{2}$ of $E$ and whose normal bundles in $M$ are isomorphic to $\pi_{1}^{*} H_{1}, \pi_{2}^{*} H_{2}$, respectively. Set $x=c_{1}\left(H_{1}\right)$ and $y=c_{1}\left(H_{2}\right)$, which are the positive generators of $H^{2}\left(\boldsymbol{C P}^{1}\right)$ and $H^{2}\left(\boldsymbol{C P ^ { 2 }}\right)$, respectively. 
Then we have

$$
c_{1}\left(\left.K_{M}^{-1}\right|_{N_{i}}\right)=c_{1}\left(\left.T M\right|_{N_{i}}\right)=c_{1}\left(T N_{i} \oplus \pi_{i}^{*} H_{i}\right)= \begin{cases}3 x+3 y & (i=1), \\ 2 x+4 y & (i=2)\end{cases}
$$

Let $\left[N_{i}\right]$ denote the fundamental cycle of $N_{i}$. Since $g^{k}$ acts on $\pi_{1}^{*} H_{1}, \pi_{2}^{*} H_{2}$ via multiplication by $\alpha^{-k}, \alpha^{k}$, respectively, it follows from $[\mathbf{1},(4.6)]$ that

$$
\begin{gathered}
\operatorname{Index}\left(D^{L}, g^{k}\right)=\left(\alpha^{-k} \mathrm{e}^{c_{1}\left(K_{M}^{-1} \mid N_{1}\right)}-1\right)^{5}\left(1-\alpha^{k} \mathrm{e}^{-c_{1}\left(\pi_{1}^{*} H_{1}\right)}\right)^{-1} \operatorname{Td}\left(T N_{1}\right)\left[N_{1}\right] \\
+\left(\alpha^{k} \mathrm{e}^{c_{1}\left(\left.K_{M}^{-1}\right|_{N_{2}}\right)}-1\right)^{5}\left(1-\alpha^{-k} \mathrm{e}^{-c_{1}\left(\pi_{2}^{*} H_{2}\right)}\right)^{-1} \mathrm{Td}\left(T N_{2}\right)\left[N_{2}\right] \\
=x y^{2} \text { coefficient of } \\
\left(\alpha^{-k} \mathrm{e}^{3 x+3 y}-1\right)^{5}\left(1-\alpha^{k} \mathrm{e}^{-x}\right)^{-1}\left(\frac{x}{1-\mathrm{e}^{-x}}\right)^{2}\left(\frac{y}{1-\mathrm{e}^{-y}}\right)^{3} \\
+\left(\alpha^{k} \mathrm{e}^{2 x+4 y}-1\right)^{5}\left(1-\alpha^{-k} \mathrm{e}^{-y}\right)^{-1}\left(\frac{x}{1-\mathrm{e}^{-x}}\right)^{2}\left(\frac{y}{1-\mathrm{e}^{-y}}\right)^{3} \\
=\left(1-\alpha^{-k}\right)\left(2 \alpha^{-k}-245 \alpha^{-2 k}+1699 \alpha^{-3 k}-2176 \alpha^{-4 k}\right) \\
+\left(1-\alpha^{-k}\right)\left(2541 \alpha^{5 k}-2034 \alpha^{4 k}+306 \alpha^{3 k}-3 \alpha^{2 k}\right) .
\end{gathered}
$$

Hence we have

$$
\begin{aligned}
& \sum_{k=1}^{p-1} \frac{1}{\alpha^{-k}-1} \operatorname{Index}\left(D^{L}, g^{k}\right) \\
& \quad \equiv 2-245+1699-2176+2541-2034+306-3=90 \quad(\bmod p),
\end{aligned}
$$

because

$$
\sum_{k=1}^{p-1} \alpha^{\mu k} \equiv-1 \quad(\bmod p)
$$

for any integer $\mu$. Therefore it follows from Proposition 2.4 that

$$
\operatorname{hol}_{\zeta}\left(\gamma_{g}\right)=\alpha^{90}
$$

which is not equal to 1 unless $p$ is a divisor of 90 . Hence it follows from Corollary 2.2 that $M$ does not admit an Einstein-Kähler metric.

\section{References}

1. M. F. Atiyah And I. M. Singer, The index of elliptic operators, III, Ann. Math. 87 (1968), 546-604.

2. M. F. Atiyah, V. K. Patodi and I. M. Singer, Spectral asymmetry and Riemannian geometry, I, Math. Proc. Camb. Phil. Soc. 77 (1975), 43-69.

3. J. M. Bismut And D. S. Freed, The analysis of elliptic families, II, Commun. Math. Phys. 107 (1986), 103-163. 
4. A. FUtAKI, An obstruction to the existence of Einstein Kähler metrics, Inventiones Math. 73 (1983), 437-443.

5. A. Futaki And S. Morita, Invariant polynomials of the automophism group of a compact complex manifold, J. Diff. Geom. 21 (1985), 135-142.

6. S. KoBAYASHI, Transformation groups in differential geometry, Classics in Mathematics (Springer, 1995).

7. K. Tsuboi, On the determinant and the holonomy of equivariant elliptic operators, Proc. Am. Math. Soc. 123 (1995), 2275-2281. 